\title{
Adsorção de boro em solos de várzea do Sul de Minas Gerais(1)
}

\begin{abstract}
Watson Rogério de Azevedo ${ }^{(2)}$, Valdemar Faquin ${ }^{(2)}$ e Luiz Arnaldo Fernandes ${ }^{(3)}$
Resumo - O objetivo deste trabalho foi avaliar a adsorção de B em quatro solos de várzea da região de Lavras, MG, por meio dos parâmetros das isotermas de Langmuir e Freundlich, no período de outubro a novembro de 1998. Amostraram-se solos Aluvial, Glei Pouco Húmico, Glei Húmico, e Orgânico artificialmente drenado, coletados na camada de 0-20 cm e peneirados para $2 \mathrm{~mm}$. Amostras com e sem calagem foram incubadas durante 30 dias. Duplicatas de $4,0 \mathrm{~g}$ de solo de cada classe foram acondicionadas em tubos de polietileno com oito diferentes doses de B $\left(0,2,4,8,12,16,24\right.$ e $\left.32 \mu \mathrm{g} \mathrm{mL}^{-1}\right)$ preparadas em $\mathrm{CaCl}_{2} \cdot 2 \mathrm{H}_{2} \mathrm{O} 0,01 \mathrm{~mol} \mathrm{~L}^{-1}$, com ácido bórico como fonte. Os teores de $\mathrm{B}$ na solução de equilíbrio foram determinados pelo método da Azometina-H. Os resultados mostraram que alto teor de matéria orgânica confere ao solo Glei Húmico maior capacidade de adsorver boro. A matéria orgânica, a superfície específica, caulinita e alumínio trocável foram os atributos dos solos que se correlacionaram diretamente com a capacidade máxima de adsorção de B (CMAB). A calagem proporcionou diminuição da CMAB em todos os solos.
\end{abstract}

Termos para indexação: disponibilidade de nutrientes, calagem, matéria orgânica.

Boron adsorption in lowland soils from Southern of the State of Minas Gerais, Brazil

\begin{abstract}
The objective of this study was to evaluate the B adsorption capacity in four lowland soils from Lavras, MG, Brazil, through Langmuir and Freundlich isotherm parameters. This study was conducted throughout October and November, 1998. Samples of Alluvial Soil, Low-Humic Gley, Humic Gley and artificially drained Bog Soil were collected in $0-20 \mathrm{~cm}$ of profundity and sieved to $2 \mathrm{~mm}$. Samples with or without liming application were incubated during 30 days. Duplicates of $4.0 \mathrm{~g}$ soil samples, conditioned in polyethylene tubes, received eight doses of $\mathrm{B}(0,2,4,8,12,16,24$ and $32 \mu \mathrm{g} \mathrm{mL}^{-1}$ ) prepared with $20 \mathrm{~mL} \mathrm{CaCl}_{2} .2 \mathrm{H}_{2} \mathrm{O} 0.01 \mathrm{~mol} \mathrm{~L}^{-1}$ solution, using boric acid. The amount of B in the balance solution was measured through the Azomethine-H method. The high organic matter content in Humic Gley soil provided higher B adsorption capacity. The adsorption was highly correlated with organic matter, specific surface area, kaolinite, and exchangeable aluminum. Lime application provided decrease in B adsorption capacity for all soils.
\end{abstract}

Index terms: nutrient availability, liming, organic matter.

\section{Introdução}

A distribuição heterogênea dos íons nos sistemas coloidais do solo é interpretada pela interação eletrostática entre seus colóides e sua fase líquida.

\footnotetext{
(1) Aceito para publicação em 5 de setembro de 2000 . Financiado pelo CNPq.

(2) Universidade Federal de Lavras, Dep. de Ciência do Solo, Caixa Postal 37, CEP 37200-000 Lavras, MG. E-mail: watsonra@ufla.br

(3) Universidade Federal de Minas Gerais, Núcleo de Ciências Agrárias, CEP 39404-006 Montes Claros, MG
}

$\mathrm{Na}$ ciência do solo, a diferença da concentração de íons entre a camada dos colóides do solo e a dupla camada difusa é denominada de adsorção (Ji, 1997).

A quantidade de B que um solo pode adsorver depende de vários fatores, tais como: concentração da solução em equilíbrio, textura do solo, tempo de contato do solo com a solução, $\mathrm{pH}$ do sistema solosolução, teor de matéria orgânica e composição mineralógica do solo, entre outros (Elrashidi \& O’Connor, 1982; Goldberg \& Glaubig, 1985; Keren et al., 1985; Yermiyahu et al., 1995). A complexidade das reações de adsorção e dados conflitantes na literatura contribuem para que os mecanismos da adsorção não sejam entendidos totalmente. 
A quantidade de um elemento adsorvido em razão da concentração remanescente na solução de equilíbrio, freqüentemente, pode ser representada por uma isoterma de adsorção (McBride, 1994). Dentre os modelos de isotermas mais utilizados estão os de Langmuir e Freundlich. Embora as equações das isotermas de adsorção sejam, freqüentemente, utilizadas na descrição da adsorção de B, elas são simplesmente relações matemáticas usadas para descrever dados, e não fornecem informações sobre os mecanismos das reações envolvidas.

A equação de Langmuir, determinada em condições de equilíbrio térmico, foi inicialmente descrita com relação à adsorção de gases por superfícies sólidas e homogêneas. A Isoterma de Langmuir supõe que a adsorção de íons, em todos os sítios da superfície, possuem a mesma atividade, e se torna máxima quando uma camada mononuclear do adsorvato cobre toda a superfície do adsorvente. Stumm (1992), no entanto, afirma que a capacidade máxima de adsorção de um íon, ajustada pela isoterma de Langmuir, não constitui evidência de que a adsorção desse íon seja um mecanismo real que computa a perda do mesmo na solução, já que, freqüentemente, a adsorção de um íon por uma superfície é seguida por interações adicionais entre outros íons na camada de adsorção.

A equação de Freundlich, desenvolvida sem base teórica, aplica-se muito bem para sólidos com propriedade de superfícies heterogêneas e, geralmente, para superfícies sólidas heterogêneas (Stumm, 1992).

A adsorção de B pelos solos tem um significado agronômico considerável, tanto na prevenção da toxidez, quanto na correção de sua deficiência no solo (Hatcher et al., 1967). Trabalhos envolvendo as isotermas de Langmuir e Freundlich procuram esclarecer o comportamento do B com relação aos mecanismos de interação com as partículas do solo e sua adsorção, levando à quantificação dos fatores que influenciam sua biodisponibilidade ( $\mathrm{Gu} \&$ Lowe, 1990; Goldberg \& Forster, 1993; Perkins, 1994; Alleoni, 1996).

O objetivo deste trabalho foi avaliar a adsorção de B em quatro solos de várzea, com e sem calagem, utilizando as isotermas de Langmuir e Freundlich.

\section{Material e Métodos}

O trabalho foi conduzido em laboratório do Departamento de Ciência do Solo da Universidade Federal de Lavras, em Lavras, MG, no período de outubro a novembro de 1998. Utilizaram-se amostras da camada superficial $(0-20 \mathrm{~cm})$ de quatro classes de solos de várzea representativos do sul de Minas Gerais: Aluvial (A), Glei Pouco Húmico (GP), Glei Húmico (GH) e Orgânico artificialmente drenado $(\mathrm{O})$; na presença e ausência de calagem. Os mesmos foram caracterizados química, física e mineralogicamente (Tabela 1).

A granulometria dos solos (areia, silte e argila) foi determinada pelo método da pipeta (Day, 1965), após queima da matéria orgânica com peróxido de hidrogênio concentrado, empregando-se $\mathrm{NaOH} 1 \mathrm{~mol} \mathrm{~L}^{-1}$ como dispersante químico e agitação rápida. Para o exame da densidade de partículas utilizou-se o método do balão

Tabela 1. Características químicas, físicas e mineralógicas dos solos (camada de 0-20 cm). Média de três repetições.

\begin{tabular}{|c|c|c|c|c|}
\hline \multirow[t]{2}{*}{ Características } & \multicolumn{4}{|c|}{ Solo $^{(1)}$} \\
\hline & A & GP & $\mathrm{GH}$ & $\mathrm{O}$ \\
\hline pH em água & 5,1 & 4,9 & 4,6 & 4,9 \\
\hline $\mathrm{P}\left(\mathrm{mg} \mathrm{kg}^{-1}\right)$ & 5 & 2 & 8 & 6 \\
\hline $\mathrm{K}\left(\mathrm{mg} \mathrm{kg}^{-1}\right)$ & 86 & 32 & 78 & 102 \\
\hline $\mathrm{B}\left(\mathrm{mg} \mathrm{kg}^{-1}\right)$ & 0,26 & 0,32 & 0,25 & 0,26 \\
\hline $\mathrm{Ca}\left(\mathrm{mmol}_{\mathrm{c}} \mathrm{dm}^{-3}\right)$ & 29 & 5 & 8 & 15 \\
\hline $\mathrm{Mg}\left(\mathrm{mmol}_{\mathrm{c}} \mathrm{dm}^{-3}\right)$ & 17 & 2 & 2 & 9 \\
\hline $\mathrm{Al}\left(\mathrm{mmol}_{\mathrm{c}} \mathrm{dm}^{-3}\right)$ & 2 & 10 & 13 & 7 \\
\hline $\mathrm{H}+\mathrm{Al}\left(\mathrm{mmol}_{\mathrm{c}} \mathrm{dm}^{-3}\right)$ & 58 & 57 & 17 & 88 \\
\hline $\mathrm{S}\left(\mathrm{mmol}_{\mathrm{c}} \mathrm{dm}^{-3}\right)$ & 48 & 9 & 12 & 28 \\
\hline CTC efetiva $\left(\mathrm{mmol}_{\mathrm{c}} \mathrm{dm}^{-3}\right)$ & 50 & 18 & 26 & 35 \\
\hline CTC a pH 7,0 $\left(\mathrm{mmol}_{\mathrm{c}} \mathrm{dm}^{-3}\right)$ & 106 & 164 & 177 & 116 \\
\hline $\mathrm{m}(\%)$ & 4 & 55 & 54 & 21 \\
\hline $\mathrm{V}(\%)$ & 44 & 12 & 7 & 24 \\
\hline Matéria orgânica $\left(\mathrm{g} \mathrm{kg}^{-1}\right)$ & 42 & 33 & 243 & 37 \\
\hline Areia grossa $\left(\mathrm{g} \mathrm{kg}^{-1}\right)$ & 0 & 30 & 9 & 0 \\
\hline Areia fina $\left(\mathrm{g} \mathrm{kg}^{-1}\right)$ & 179 & 611 & 230 & 120 \\
\hline Silte $\left(\mathrm{g} \mathrm{kg}^{-1}\right)$ & 471 & 179 & 391 & 570 \\
\hline Argila $\left(\mathrm{g} \mathrm{kg}^{-1}\right)$ & 350 & 180 & 370 & 310 \\
\hline S. específica $\left(\mathrm{m}^{2} \mathrm{~g}^{-1}\right)$ & 137,4 & 120,8 & 334,6 & 180,7 \\
\hline Densidade do solo $\left(\mathrm{g} \mathrm{cm}^{-3}\right)$ & 0,76 & 1,05 & 0,40 & 0,64 \\
\hline Ferro ditionito $\left(\mathrm{g} \mathrm{kg}^{-1}\right)$ & 12,3 & 1,8 & 2,3 & 5,3 \\
\hline $\mathrm{Fe}_{2} \mathrm{O}_{3}\left(\mathrm{~g} \mathrm{~kg}^{-1}\right)$ & 74,5 & 13,0 & 13,3 & 42,8 \\
\hline $\mathrm{SiO}_{2}\left(\mathrm{~g} \mathrm{~kg}^{-1}\right)$ & 209,3 & 84,2 & 126,1 & 243,4 \\
\hline $\mathrm{Al}_{2} \mathrm{O}_{3}\left(\mathrm{~g} \mathrm{~kg}^{-1}\right)$ & 221,1 & 112,2 & 175,5 & 247,1 \\
\hline $\mathrm{TiO}_{2}\left(\mathrm{~g} \mathrm{~kg}^{-1}\right)$ & 9,3 & 6,2 & 5,5 & 7,3 \\
\hline Caulinita (Ct) $\left(\mathrm{g} \mathrm{kg}^{-1}\right)$ & 143,4 & 64,9 & 48,2 & 120,5 \\
\hline Gibbsita (Gb) $\left(\mathrm{g} \mathrm{kg}^{-1}\right)$ & 48,6 & 19,0 & 53,0 & 57,4 \\
\hline $\mathrm{Ct} /(\mathrm{Ct}+\mathrm{Gb})$ & 0,74 & 0,77 & 0,48 & 0,67 \\
\hline $\mathrm{ki}$ & 1,61 & 1,28 & 1,22 & 1,67 \\
\hline
\end{tabular}

${ }^{(1)}$ A: Aluvial; GP: Glei Pouco Húmico; GH: Glei Húmico; O: Orgânico artificialmente drenado. 
volumétrico com álcool etílico (Embrapa, 1997), a densidade do solo foi determinada através do método do anel volumétrico (Blake, 1965) e a superfície específica foi determinada pelo método do etileno-glicol-monoetil-éter (Heilman et al., 1965). A caracterização química (pH em água, $\mathrm{P}, \mathrm{Ca}, \mathrm{Mg}, \mathrm{K}, \mathrm{H}+\mathrm{Al}$ e $\mathrm{Al}$ ) e matéria orgânica do solo foram determinadas conforme Embrapa (1997). O B foi extraído em água quente (Berger \& Truog, 1939) e quantificado pelo método da Azometina-H (Wolf, 1974). Os teores totais dos óxidos $\left(\mathrm{SiO}_{2}, \mathrm{Al}_{2} \mathrm{O}_{3}, \mathrm{Fe}_{2} \mathrm{O}_{3}\right.$ e $\left.\mathrm{TiO}_{2}\right)$ por ataque sulfúrico, segundo Vettori (1969), com modificações propostas pela Embrapa (1997). A fração argila foi tratada com ditionito-citrato-bicarbonato de sódio (DCB) (Mehra \& Jackson, 1960). Na fração argila desferrificada, foram quantificados os teores de caulinita e gibbsita, através da análise termodiferencial (ATD). A composição dessa mesma fração foi determinada por difração de raios X (Figura 1).

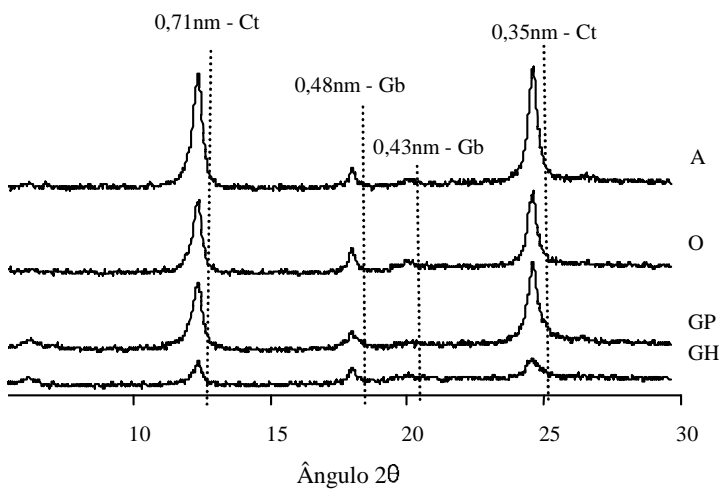

Figura 1. Difratogramas de raios $X$ da fração argila desferrificada, saturada com sódio, à temperatura ambiente e lâminas orientadas, no horizonte $\mathrm{A}$ dos solos de várzea. Caulinita (Ct), gibbsita (Gb), Aluvial (A), Glei Pouco Húmico (GP), Glei Húmico (GH) e Orgânico (O).
A calagem constou da aplicação de $\mathrm{CaCO}_{3}$ e $\mathrm{MgCO}_{3}$ p.a., na relação equivalente $\mathrm{Ca}: \mathrm{Mg}$ de $4: 1$, determinada por curva de incubação em ensaio prévio realizado em laboratório. As doses estabelecidas, visando elevar a saturação por bases $(\mathrm{V})$ a $70 \%$ devido à utilização de amostras em outro experimento com rabanete e doses de $\mathrm{B}$, foram de 4,4, 2,7, 7,8 e 3,5 $\mathrm{t} \mathrm{ha}^{-1}$, nos solos A, GP, GH e O, respectivamente. As amostras com e sem calagem foram incubadas por 30 dias. Alguns resultados dos atributos dos solos, antes e após a calagem, se encontram na Tabela 2.

Para a quantificação de B adsorvido pelos solos utilizou-se uma adaptação, em relação às concentrações utilizadas pelo método preconizado por Okazaki \& Chao (1968). Foram utilizadas amostras de 4,0 g de solo (TFSE), acondicionadas em tubos de polietileno, contendo $20 \mathrm{~mL}$ de uma solução de $\mathrm{CaCl}_{2} 0,01 \mathrm{~mol} \mathrm{~L}^{-1}$ com diferentes concentrações de boro $\left(0,2,4,8,12,16,24\right.$ e $\left.32 \mu \mathrm{g} \mathrm{mL}^{-1}\right)$, usando-se ácido bórico como fonte; foram utilizadas duas repetições de cada concentração. Segundo Alleoni (1996), a utilização de concentrações mais baixas é mais adequada para representar a quantidade de B contida nos solos brasileiros. Assim, considerando a massa de solo utilizada, obtiveram-se: $0,10,20,40,60,80,120$ e $160 \mu \mathrm{g} \mathrm{g}^{-1} \mathrm{~B}$ no solo.

Após a agitação por 24 horas, a suspensão foi centrifugada por quinze minutos a $2.000 \mathrm{rpm}$ (Shumway \& Jones, 1972), e uma alíquota de $2 \mathrm{~mL}$ foi retirada do sobrenadante e colocada em balão volumétrico, completando-se o volume para $50 \mathrm{~mL}$. O B nessa solução foi quantificado pelo método da Azometina-H.

A quantidade de B adsorvido em cada solo foi obtida pela diferença entre a concentração de $\mathrm{B}$ na solução inicial e o B remanescente na solução de equilíbrio após a agitação e centrifugação. Foi descontado o B presente na dose zero para efeito de cálculos da quantidade de $\mathrm{B}$ adsorvido.

$\mathrm{O} B$ adsorvido $(\mathrm{x} / \mathrm{m})$ foi plotado em função do B remanescente nas soluções de equilíbrio (C) para obtenção do ajuste da curva de adsorção, nas suas formas lineares,

Tabela 2. Resultados analíticos de pH, Al trocável, capacidade de troca catiônica (CTC) efetiva, capacidade de troca catiônica a pH 7,0, saturação por bases $(\mathrm{V})$ e matéria orgânica das amostras antes e após a calagem. Média de três repetições.

\begin{tabular}{|c|c|c|c|c|c|c|}
\hline Solo $^{(1)}$ & $\mathrm{pH}\left(\mathrm{H}_{2} \mathrm{O}\right)$ & $\begin{array}{c}\mathrm{Al} \\
---- \\
\end{array}$ & $\begin{array}{l}\text { CTC efetiva } \\
--\left(\mathrm{mmol}_{\mathrm{c}} \mathrm{dm}^{-3}\right.\end{array}$ & CTC a pH 7,0 & $\begin{array}{c}\mathrm{V} \\
(\%) \\
\end{array}$ & $\begin{array}{c}\text { Matéria orgânica } \\
\qquad\left(\mathrm{g} \mathrm{kg}^{-1}\right)\end{array}$ \\
\hline & \multicolumn{6}{|c|}{ Sem calagem } \\
\hline A & 5,1 & 2 & 50 & 106 & 44 & 42 \\
\hline GP & 4,9 & 10 & 18 & 164 & 12 & 33 \\
\hline GH & 4,6 & 13 & 26 & 177 & 7 & 243 \\
\hline \multirow[t]{2}{*}{$\mathrm{O}$} & 4,9 & 7 & 35 & 116 & 24 & 37 \\
\hline & \multicolumn{6}{|c|}{ Com calagem } \\
\hline A & 6,6 & 0 & 85 & 108 & 79 & 40 \\
\hline GP & 6,0 & 1 & 86 & 163 & 63 & 30 \\
\hline GH & 5,6 & 1 & 86 & 163 & 62 & 237 \\
\hline $\mathrm{O}$ & 5,9 & 0 & 72 & 128 & 60 & 35 \\
\hline
\end{tabular}


de Langmuir $[\mathrm{C} /(\mathrm{x} / \mathrm{m})$ em função de $\mathrm{C}]$ e Freundlich $[\log (\mathrm{x} / \mathrm{m})$ em função de $\log \mathrm{C}]$.

\section{Resultados e Discussão}

Quanto às características químicas, físicas e mineralógicas dos solos (Tabela 1), verifica-se que os solos são bastante heterogêneos, predominantemente ácidos e distróficos $(\mathrm{V}<50 \%)$ e apresentam uma grande variação textural, onde um maior teor de matéria orgânica no solo GH implicou um solo de menor densidade (Ds) e alta superfície específica.

$\mathrm{O}$ resultado da análise mineralógica (Tabela 1) mostra que os solos A, GP e O possuem mineralogia mais caulinítica, razão caulinita/(caulinita + gibbsita) $>0,5$, em contraste com o solo $\mathrm{GH}$, o qual possui mineralogia mais gibbsítica, razão caulinita/ (caulinita + gibbsita) $<0,5$. Os valores de $\mathrm{Fe}_{2} \mathrm{O}_{3}$ e $\mathrm{Fe}_{\mathrm{d}}$ nos solos GP e GH mostram a baixa quantidade de Fe total e Fe livre no sistema, característica principal dos solos Glei, que possuem um ambiente de intensa redução de $\mathrm{Fe}$ e cores neutras da matriz do solo (Oliveira et al., 1992). Os valores observados no índice ki, menores que 2,0, além de mostrar a presença de gibbsita, indicam que os materiais que formaram esses solos possuem alto estágio de meteorização (Moniz, 1972). Os difratogramas de raios X obtidos da fração argila (Figura 1), após o tratamento com ditionito-citrato-bicarbonato de sódio, revelaram a presença de caulinita e gibbsita nos horizontes A de todos os solos estudados, manifestado pela presença dos picos 0,71 e $0,35 \mathrm{~nm}$ na caulinita e $0,48 \mathrm{~nm}$ e $0,43 \mathrm{~nm}$ na gibbsita. Nos solos A, GP e O, os picos da caulinita são mais bem expressos, indicando maiores teores, fato confirmado pela análise termodiferencial (Tabela 1).

$\mathrm{O}$ valor do $\mathrm{pH}$ aumentou em todos os solos após a calagem, mas não atingiu a faixa alcalina, ou seja, pH maior que sete e menor ou igual a dez (Tabela 2). Nessa faixa, há um aumento da concentração de $\mathrm{B}(\mathrm{OH})_{4}^{-}$, que possui maior afinidade com os óxidos de Fe e Al (Goldberg \& Glaubig, 1985) e matéria orgânica (Yermiyahu et al., 1995). Segundo Evans (1987), a adsorção de B pelos óxidos e pela matéria orgânica é efetivamente relevante em $\mathrm{pH}$ na faixa alcalina, atingindo a máxima adsorção a pH 9,0.
As quantidades de B adsorvido aumentaram com as quantidades adicionadas, ao passo que a porcentagem adsorvida em relação à quantidade adicionada diminuiu com o aumento da concentração de B na solução de equilíbrio (Tabela 3 ). O aumento relativamente menor, à medida que as soluções ficam mais concentradas, concorda com os resultados observados por Correa et al. (1985) e Valladares et al. (1998). Tal fato provavelmente ocorre pela não-saturação da superfície adsorvente em sistemas heterogêneos (Goldberg et al., 1996).

A partir dos dados de B adsorvido $\left(\mu \mathrm{g} \mathrm{g}^{-1}\right)$ e B remanescente na solução de equilíbrio $\left(\mu \mathrm{g} \mathrm{mL}^{-1}\right)$, foram obtidos os parâmetros das isotermas de Langmuir \& Freundlich (Tabela 4). As constantes $\mathrm{k} \mathrm{e} \mathrm{b}$ das isotermas, obtidas pelos coeficientes das equações apresentadas na Tabela 4, encontram-se na Tabela 5.

A capacidade máxima de adsorção de $\mathrm{B}$ (CMAB) (constante $\mathrm{b}$ de Langmuir) nos quatro solos estudados, com e sem calagem, foi, em ordem decrescente, $\mathrm{GH}>\mathrm{GP}>\mathrm{O}>\mathrm{A}$ (Tabela 5). Nota-se, em todos os solos, uma diminuição da CMAB quando se aplicou o calcário. Essa diminuição, em termos relativos, foi de aproximadamente 19, 30, 23 e 26\% nos solos A, GP, GH e O, respectivamente. Resultados semelhantes foram observados por Olson \& Berger (1946) e Mezuman \& Keren (1981). Com a calagem, a maioria dos sítios de adsorção, representados pelos grupos carboxílicos dos ácidos húmicos da matéria orgânica, provavelmente foram ocupados pelo íon $\mathrm{Ca}^{2+}$, ou seja, poucos sítios ficaram disponíveis para adsorver B (Gu \& Lowe, 1990). Esses mesmos autores afirmam que o íon $\mathrm{Ca}^{2+}$ possui maior afinidade pelos grupos carboxílicos que o boro.

Na Tabela 6 são apresentadas as quantidades médias de B adsorvido pelos solos estudados, independentemente da dose aplicada, na ausência e na presença de calagem. Verifica-se que não houve diferença na quantidade de $\mathrm{B}$ adsorvido, entre os solos, sem a aplicação da calagem. No entanto, com a calagem, o solo GH apresentou a maior quantidade de $\mathrm{B}$ adsorvido devido ao seu alto teor de matéria orgânica, que confere maior valor da superfície específica, podendo-se inferir que esse solo apresenta maior quantidade de sítios de adsorção de boro.

Em todos os solos, os valores estimados da CMAB (Tabela 5) foram maiores que os obtidos após a adi- 
ção da maior dose de B utilizada, à exceção do solo A sem calagem (Tabela 3). Os valores da CMAB podem ter sido super ou subestimados pelo modelo de Langmuir, como também foi verificado em trabalhos com outros tipos de solos (Goldberg \& Forster, 1993; Alleoni, 1996; Valladares et al., 1998). No entanto, os valores estimados da CMAB obtidos neste trabalho não podem ser comparados com os dos outros solos. Para estabelecer uma comparação, deve-se considerar o aspecto qualitativo da estimativa, o que só é possível quando se comparam solos de uma mesma região que não diferem entre si quanto à sua mineralogia (Cunha, 1989).

O fenômeno de adsorção de B pelos solos é grandemente afetado pelos seus atributos físicos, químicos e mineralógicos (Keren et al., 1985). Nes-

Tabela 3. Quantidades de B adsorvido e porcentagem de adsorção em quatro solos de várzea sem e com calagem, em função de B adicionado.

\begin{tabular}{|c|c|c|c|c|c|c|c|c|}
\hline \multirow{3}{*}{$\begin{array}{l}\mathrm{B} \text { adicionado } \\
\left(\mu \mathrm{g} \mathrm{g}^{-1}\right)\end{array}$} & \multicolumn{8}{|c|}{$\mathrm{B}$ adsorvido no solo ${ }^{(1)}$} \\
\hline & \multicolumn{2}{|c|}{ A } & \multicolumn{2}{|c|}{ GP } & \multicolumn{2}{|c|}{$\mathrm{GH}$} & \multicolumn{2}{|c|}{$\mathrm{O}$} \\
\hline & $\left(\mu \mathrm{g} \mathrm{g}^{-1}\right)$ & $(\%)$ & $\left(\mu \mathrm{g} \mathrm{g}^{-1}\right)$ & $(\%)$ & $\left(\mu g^{-1}\right)$ & $(\%)$ & $\left(\mu g^{-1}\right)$ & $(\%)$ \\
\hline & \multicolumn{8}{|c|}{ Sem calagem } \\
\hline 10 & 7,45 & 74 & 4,12 & 41 & 4,76 & 48 & 4,58 & 46 \\
\hline 20 & 8,80 & 44 & 9,10 & 45 & 12,38 & 62 & 9,20 & 46 \\
\hline 40 & 12,81 & 32 & 13,06 & 33 & 16,67 & 41 & 10,60 & 26 \\
\hline 60 & 11,83 & 20 & 16,16 & 27 & 18,84 & 31 & 12,50 & 21 \\
\hline 80 & 13,02 & 16 & 17,55 & 22 & 24,14 & 30 & 15,86 & 20 \\
\hline 120 & 16,33 & 14 & 19,40 & 16 & 23,73 & 20 & 15,56 & 14 \\
\hline \multirow[t]{2}{*}{160} & 19,89 & 12 & 21,30 & 13 & 27,55 & 17 & 17,89 & 11 \\
\hline & \multicolumn{8}{|c|}{ Com calagem } \\
\hline 10 & 2,86 & 29 & 7,43 & 74 & 7,26 & 73 & 3,57 & 36 \\
\hline 20 & 7,09 & 35 & 8,31 & 41 & 16,38 & 82 & 6,94 & 35 \\
\hline 40 & 10,07 & 25 & 10,40 & 26 & 15,01 & 37 & 8,55 & 21 \\
\hline 60 & 10,29 & 17 & 11,60 & 19 & 15,26 & 25 & 9,76 & 16 \\
\hline 80 & 12,48 & 16 & 14,36 & 18 & 19,72 & 25 & 9,97 & 12 \\
\hline 120 & 12,92 & 11 & 14,02 & 12 & 23,04 & 19 & 13,58 & 11 \\
\hline 160 & 13,36 & 8 & 15,81 & 10 & 22,85 & 14 & 13,81 & 9 \\
\hline
\end{tabular}

(1)A: Aluvial; GP: Glei Pouco Húmico; GH: Glei Húmico; O: Orgânico artificialmente drenado.

Tabela 4. Equações de regressão e coeficiente de determinação $\left(\mathrm{R}^{2}\right)$ para as isotermas de Langmuir e Freundlich obtidas a partir de oito doses de $\mathrm{B}$ em $\mathrm{CaCl}_{2} 0,01 \mathrm{~mol} \mathrm{~L}^{-1}$, em quatro solos de várzea, sem e com calagem.

\begin{tabular}{|c|c|c|c|c|}
\hline \multirow[t]{2}{*}{$\mathrm{Solo}^{(1)}$} & \multicolumn{2}{|l|}{ Langmuir } & \multicolumn{2}{|c|}{ Freundlich } \\
\hline & Equação & $\mathrm{R}^{2}$ & Equação & $\mathrm{R}^{2}$ \\
\hline & \multicolumn{4}{|c|}{ Sem calagem } \\
\hline A & $\mathrm{Y}=0,121+0,055^{* *} \mathrm{X}$ & 0,93 & $\mathrm{Y}=0,907+0,219 * * \mathrm{X}$ & 0,86 \\
\hline GP & $Y=0,141+0,044 * * X$ & 0,96 & $\mathrm{Y}=0,893+0,309 * \mathrm{X}$ & 0,94 \\
\hline GH & $Y=0,159+0,032 * * X$ & 0,94 & $Y=0,662+0,629 * x$ & 0,93 \\
\hline \multirow[t]{2}{*}{$\mathrm{O}$} & $\mathrm{Y}=0,168+0,054 * * \mathrm{X}$ & 0,96 & $\mathrm{Y}=0,753+0,345 * * \mathrm{X}$ & 0,88 \\
\hline & \multicolumn{4}{|c|}{ Com calagem } \\
\hline A & $Y=0,198+0,068 * * X$ & 0,97 & $\mathrm{Y}=0,767+0,263 * * \mathrm{X}$ & 0,83 \\
\hline GP & $\mathrm{Y}=0,112+0,063 * * \mathrm{X}$ & 0,98 & $Y=0,895+0,195 * x$ & 0,84 \\
\hline GH & $\mathrm{Y}=0,087+0,042 * * \mathrm{X}$ & 0,97 & $\mathrm{Y}=0,899+0,336 * x$ & 0,96 \\
\hline $\mathrm{O}$ & $Y=0,304+0,064 * * X$ & 0,97 & $\mathrm{Y}=0,713+0,286 * * \mathrm{X}$ & 0,94 \\
\hline
\end{tabular}

(1)A: Aluvial; GP: Glei Pouco Húmico; GH: Glei Húmico; O: Orgânico artificialmente drenado. **Significativo a 1\% de probabilidade. 
te trabalho, a interação solo-B foi verificada ao se estabelecerem correlações entre a CMAB e os atributos dos solos.

Os valores estimados da CMAB não se correlacionaram significativamente com o $\mathrm{pH}$ dos solos. Tal fato deve-se, provavelmente, aos valores atingidos de $\mathrm{pH}$, mesmo com a calagem (Tabela 2), abaixo da faixa alcalina, como discutido anteriormente. Na faixa ácida há predominância de ácido bórico $\left(\mathrm{H}_{3} \mathrm{BO}_{3}\right)$, em relação ao íon borato $\left[\mathrm{B}(\mathrm{OH})_{4}{ }^{-}\right]$, que não é afetado pela reação de adsorção (Gu \& Lowe, 1990). Entretanto, a adsorção pode ocorrer em condições ácidas e ambientes heterogêneos como o solo. Assim, os valores de adsorção encontrados nesse trabalho, deram-se, provavelmente, por mecanismos não dependentes de $\mathrm{pH}$.

Os teores de matéria orgânica dos solos se correlacionaram significativamente com a $\mathrm{CMAB}$ $\left(\mathrm{r}=0,82^{* *}\right)$, mostrando que a matéria orgânica, provavelmente, é um dos sítios mais ativos de adsorção de B. Parks \& White (1952) relatam que $\mathrm{H}^{+}$ligados a ácidos húmicos, em $\mathrm{pH}$ abaixo de 6,5 , retêm grande quantidade de boro.

A correlação significativa com a superfície específica $\left(r=0,76^{*}\right)$ sugere que, quanto maior o valor

Tabela 5. Constantes de Langmuir e Freundlich obtidas a partir dos resultados verificados pela linearização das respectivas isotermas, em quatro solos de várzea, com e sem calagem.

\begin{tabular}{|c|c|c|c|c|}
\hline \multirow[t]{2}{*}{ Solo ${ }^{(1)}$} & \multirow[t]{2}{*}{ Calagem } & \multicolumn{2}{|c|}{ Langmuir } & \multirow{2}{*}{$\frac{\text { Freundlich }}{\mathrm{k}\left(\mathrm{mL} \mu \mathrm{g}^{-1}\right)}$} \\
\hline & & $\mathrm{k}\left(\mathrm{mL} \mu \mathrm{g}^{-1}\right)$ & $\begin{array}{c}\mathrm{b}(\mathrm{CMAB})^{(2)} \\
\left(\mu \mathrm{g} \mathrm{g}^{-1}\right)\end{array}$ & \\
\hline \multirow[t]{2}{*}{ A } & Sem & 0,4545 & 18,1818 & 8,0723 \\
\hline & Com & 0,3434 & 14,7059 & 5,8479 \\
\hline \multirow[t]{2}{*}{ GP } & Sem & 0,3121 & 22,7272 & 7,8163 \\
\hline & Com & 0,5625 & 15,8730 & 7,8523 \\
\hline \multirow[t]{2}{*}{$\mathrm{GH}$} & Sem & 0,2012 & 30,9597 & 4,5919 \\
\hline & Com & 0,4827 & 23,8095 & 7,9250 \\
\hline \multirow[t]{2}{*}{$\mathrm{O}$} & Sem & 0,3214 & 18,5185 & 5,6623 \\
\hline & Com & 0,2105 & 15,6248 & 5,1642 \\
\hline
\end{tabular}

(1)A: Aluvial; GP: Glei Pouco Húmico; GH: Glei Húmico; O: Orgânico artificialmente drenado. ${ }^{(2)} \mathrm{CMAB}$ : capacidade máxima de adsorção de boro.

Tabela 6. Quantidade de B ( $\left.\mu \mathrm{g} \mathrm{g}^{-1}\right)$ adsorvida em quatro solos de várzea, com e sem calagem $^{(1)}$.

\begin{tabular}{lrrrr}
\hline Calagem & \multicolumn{1}{c}{$\mathrm{A}$} & \multicolumn{1}{c}{$\mathrm{GP}$} & $\mathrm{GH}$ & \multicolumn{1}{c}{$\mathrm{O}$} \\
\hline Sem & $11,23 \mathrm{aA}$ & $12,60 \mathrm{aA}$ & $15,70 \mathrm{aA}$ & $10,66 \mathrm{aA}$ \\
Com & $8,58 \mathrm{bA}$ & $10,22 \mathrm{abA}$ & $14,79 \mathrm{aA}$ & $8,16 \mathrm{bA}$ \\
\hline
\end{tabular}

${ }^{(1)}$ A: Aluvial; GP: Glei Pouco Húmico; GH: Glei Húmico; O: Orgânico artificialmente drenado; médias seguidas da mesma letra, minúscula na linha e maiúscula na coluna, não diferem entre si a $5 \%$ de probabilidade, pelo teste de Tukey. desse atributo do solo, maior a possibilidade da existência de sítios de adsorção de boro. Nos solos ora estudados, a superfície específica é bastante afetada pelo teor de matéria orgânica e a fração mineralógica de composição, bastante oxídica (Tabela 1).

Estabeleceu-se uma correlação significativa entre o teor de caulinita presente nos solos de várzea $\mathrm{e}$ a CMAB $\left(r=-0,71^{*}\right)$. Esse resultado indica baixa reatividade da caulinita, talvez devido à sua menor superfície específica, ou menor número de sítios reativos que adsorvem boro. Neste caso, o fenômeno de adsorção se dá, provavelmente, por adsorção específica entre o B e a superfície da caulinita. Goldberg et al. (1993), estudando a adsorção de B em várias superfícies, mostraram que a adsorção de B na caulinita foi dependente de uma pequena energia iônica em relação à adsorção máxima, sugerindo a formação de um complexo de esfera interna, ou seja, a adsorção de B se deu diretamente na superfície da caulinita, sem a presença de moléculas de água.

Observou-se uma correlação significativa entre a CMAB e o teor de Al trocável dos solos ora estudados $\left(r=0,65^{*}\right)$, o que mostra que quanto maior o teor de $\mathrm{Al}$ trocável em um solo, maior a quantidade de $\mathrm{B}$ adsorvida por ele. Tal fato pode ocorrer devido à potencial formação e precipitação de hidróxido de alumínio, com a aplicação de calcário. Segundo Hatcher et al. (1967), o hidróxido de alumínio adsorve grandes quantidades de boro.

\section{Conclusões}

1. De modo geral, maior teor de matéria orgânica no solo confere maior capacidade máxima de adsorção de boro.

2. A adsorção de B correlaciona-se diretamente com matéria orgânica, superfície específica, caulinita, e Al trocável.

3. A calagem diminui a capacidade máxima de adsorção de B nos solos de várzea.

\section{Referências}

ALLEONI, L. R. F. Adsorção de boro em Podzólico e Latossolos paulistas. Piracicaba : ESALQ, 1996. 127 p. Tese de Doutorado.

BERGER, K. C.; TRUOG, E. Boron determination in soils and plants. Industrial and Engineering Chemistry, Washington, v. 11, p. 540-545, 1939. 
BLAKE, G. R. Bulk density. In: BLACK, C. A. (Ed.). Methods of soil analysis: physical and mineralogical properties including statistics of measurement and sampling. Madison : American Society of Agronomy, 1965. p. 374-390.

CORREA, A. E.; PAVAN, M. A.; MIYAZAWA, M. Aplicação de boro no solo e respostas do cafeeiro. Pesquisa Agropecuária Brasileira, Brasília, v. 20, n. 2, p. 177-181, fev. 1985.

CUNHA, R. C. A. Retenção e movimento de zinco em solos do Estado de São Paulo. Piracicaba : ESALQ, 1989. 115 p. Dissertação de Mestrado.

DAY, P. R. Particle fractionation and particle-size analysis. In: BLACK, C. A. (Ed.). Methods of soil analysis: physical and mineralogical properties including statistics of measurement and sampling. Madison : American Society of Agronomy, 1965. p. 545-566.

ELRASHIDI, M. A.; O'CONNOR, G. A. Boron sorption and desorption in soils. Soil Science Society of America Journal, Madison, v. 46, n. 1, p. 27-31, 1982.

EMBRAPA. Centro Nacional de Pesquisa de Solos (Rio de Janeiro, RJ). Manual de métodos e análises de solos. 2. ed. Rio de Janeiro : Embrapa-CNPS, 1997. 212 p. (Embrapa-CNPS. Documentos, 1)

EVANS, L. J. Retention of boron by agricultural soils from Ontario. Canadian Journal of Soil Science, Ottawa, v. 67, p. 33-42, 1987.

GOLDBERG, S.; FORSTER, H. S. Boron sorption on calcareous soils and reference calcites. Soil Science, Baltimore, v. 152, n. 4, p. 304-310, 1993.

GOLDBERG, S.; FORSTER, H. S.; HERCK, E. L. Boron adsorption mechanisms on oxides, clay minerals and soils inferred from ionic strength effects. Soil Science Society of America Journal, Madison, v. 57, p. 125-128, 1993.

GOLDBERG, S.; FORSTER, H. S.; LESCH, S. M.; HERCK, E. L. Influence of anion competition on boron adsorption by clays and soils: boron adsorption mechanisms on oxides, clay minerals and soils. Soil Science, Baltimore, v. 161, n. 2, p. 99-103, 1996.

GOLDBERG, S.; GLAUBIG, R. A. Boron adsorption on aluminum and iron oxide minerals. Soil Science Society of America Journal, Madison, v. 49, p. 1374-1379, 1985.

GU, B.; LOWE, L. E. Studies on the adsorption of boron on humic acids. Canadian Journal of Soil Science, Ottawa, v. 70, p. 305-311, 1990.
HATCHER, J. T.; BOWER, C. A.; CLARK, M. Adsorption of boron by soils as influenced by hidroxy aluminum and surface area. Soil Science, Baltimore, v. 104, p. 422-426, 1967.

HEILMAN, M. D.; CARTER, D. L.; GONZALES, C. L. The ethylene glycol monoethyl ether (EMEG) technique for determining soil surface area. Soil Science, Baltimore, v. 100, p. 409-413, 1965.

JI, G. L. Electrostatic adsorption of anions. In: YU, T. R. (Ed.). Chemistry of variable charge soils. New York : Oxford University Press, 1997. p. 112-139.

KEREN, R.; BINGHAM, F. T.; RHOADS, J. D. Plant uptake of boron as affected by boron distribution between liquid and solid phases in soil. Soil Science Society of America Journal, Madison, v. 9, p. 297-302, 1985.

McBRIDE, M. B. Environmental chemistry of soils. New York : Oxford University Press, 1994. 406 p.

MEHRA, O. P.; JACKSON, N. L. Iron oxide removal from soils and clays by a dithionite-citrate system buffered with sodium bicarbonate. Clays and Clay Minerals, Clarkson, v. 3, p. 317-327, 1960.

MEZUMAN, U.; KEREN, R. Boron adsorption by soils using a phenomenological adsorption equation. Soil Science Society of America Journal, Madison, v. 45, p. $722-726,1981$.

MONIZ, A. C. Mineralogia do solo. In: MONIZ, A. C. (Ed.). Elementos de pedologia. São Paulo : Polígono/USP, 1972. p. 391-408.

OKAZAKI, E.; CHAO, T. T. Boron adsorption and desorption by some Hawaiian soils. Soil Science, Baltimore, v. 105, p. 255-259, 1968.

OLIVEIRA, J. B.; JACOMINE, P. K.; CAMARGO, M. N. Classes de solos do Brasil: guia auxiliar para seu reconhecimento. 2. ed. Jaboticabal : FUNEP, 1992. 201 p.

OLSON, R. V.; BERGER, K. C. Boron fixation as influenced by $\mathrm{pH}$, organic matter content and other factors. Soil Science Society of America Proceedings, Madison, v. 11, p. 216-220, 1946.

PARKS, W. L.; WHITE, J. L. Boron retention by clay and humus systems saturated whit various cations. Soil Science Society of America Proceedings, Madison, v. 16, p. 298330, 1952.

PERKINS, P. V. Mathematical modeling of soil boron concentration under field moisture conditions using the Langmuir and Freundlich adsorption isotherms. 
Communications in Soil Science and Plant Analysis, New York, v. 25, n. 15/16, p. 2649-2664, 1994.

SHUMWAY, J. S.; JONES, J. P. Boron adsorption isotherm: a method to estimate boron fertilizer requirement. Communications in Soil Science and Plant Analysis, New York, v. 3, p. 477-485, 1972.

STUMM, W. Chemistry on the solid-water interface. New York : J. Wiley, 1992. 428 p.

VALLADARES, G. S.; PEREIRA, M. G.; ALVES, G. C. Aplicação de duas isotermas de adsorção de boro em solos de baixada do Estado do Rio de Janeiro. Revista Brasileira de Ciência do Solo, Campinas, v. 22, p. 361-365, 1998.

VETTORI, L. Métodos de análise de solo. Rio de Janeiro : Ministério da Agricultura, 1969. 24 p. (Boletim Técnico, 7).

WOLF, B. Improvement in the azomethine-H method for the determination of boron. Communications in Soil Science and Plant Analysis, New York, v. 5, p. 39-44, 1974.

YERMIYAHU, U.; KEREN, R.; CHEN, Y. Boron sorption by soil in the presence of composted organic matter. Soil Science Society of America Journal, Madison, v. 59, p. 405-409, 1995. 